\title{
Fatores de risco para retinopatia da prematuridade: revisão integrativa
}

\author{
Francisco Alysson Costa Souza ${ }^{1}$ \\ Jéssica Naiara de Medeiros Araújo ${ }^{2}$ \\ Raffaela Patricia da Silva Soares ${ }^{3}$ \\ Marina Marisa Palhano dos Santos ${ }^{4}$ \\ Marcos Antonio Ferreira Júnior ${ }^{5}$ \\ Allyne Fortes Vitor 6
}

\section{RESUMO}

Sumarizar os fatores de risco para a retinopatia da prematuridade em recém-nascidos internados em unidade de terapia intensiva neonatal a partir de estudos primários publicados em bases de dados. Trata-se de uma revisão integrativa realizada nas seguintes bases: SCOPUS, PubMed, Science Direct, Web of Science e CINAHL. A amostra final foi constituída por 26 artigos. Elencou-se categorias temáticas com os fatores de risco da retinopatia da prematuridade, a saber: fatores relacionados à terapêutica clínica, ao uso de medicamentos, comorbidades relacionadas à retinopatia da prematuridade, características neonatais, características clínicas associadas à retinopatia da prematuridade e fatores maternos. Conhecer os fatores de risco implica na detecção precoce da retinopatia mediante adoção de medidas preventivas como monitoramento da oxigenoterapia, ventilação mecânica e transfusões sanguíneas, além da avaliação das características neonatais, de maneira a permitir uma triagem oftalmológica que impeça o desenvolvimento da doença, suas sequelas e/ou viabilize tratamento na fase inicial da doença.

Descritores: Fatores de Risco; Retinopatia da Prematuridade; Unidade de Terapia Intensiva Neonatal.

\footnotetext{
${ }^{1}$ Enfermeiro. Natal, RN, Brasil. E-mail: alysson_med@yahoo.com.br.

2 Enfermeira, Mestre em Enfermagem. Discente de Doutorado do Programa de Pós-Graduação em Enfermagem da Universidade Federal do Rio Grande do Norte. Natal, RN, Brasil. E-mail: jessicanaiara rn@hotmail.com.

${ }^{3}$ Discente do curso de Graduação em Enfermagem da Universidade Federal do Rio Grande do Norte. Bolsista de Iniciação Científica da Universidade Federal do Rio Grande do Norte. Natal, RN, Brasil. E-mail: raffaelapatricia@hotmail.com.

${ }^{4}$ Discente do curso de Graduação em Enfermagem da Universidade Federal do Rio Grande do Norte. Bolsista de Iniciação Científica da Universidade Federal do Rio Grande do Norte. Natal, RN, Brasil. E-mail: marina.palhano@yahoo.com.br.

${ }^{5}$ Enfermeiro, Doutor em Saúde e Desenvolvimento na Região Centro-Oeste. Professor Adjunto da Universidade Federal de Mato Grosso do Sul.

Campo Grande, MS, Brasil. E-mail: marcos_nurse@hotmail.com.

${ }^{6}$ Enfermeira, Doutora em Enfermagem. Professora Adjunta da Universidade Federal do Rio Grande do Norte. Natal, RN, Brasil. E-mail: allynefortes@yahoo.com.br.
}

Artigo recebido: 01/11/2016.

Artigo aprovado: 08/12/2017.

Artigo publicado: $17 / 04 / 2018$.

\section{Como citar esse artigo:}

Souza FAC, Araújo JNM, Soares RPS, Santos MMP, Ferreira Júnior MA, Vitor AF. Fatores de risco para retinopatia da prematuridade: revisão integrativa. Rev. Eletr. Enf. [Internet] 2018; [acesso em: ]20:v20a04. Disponível em: http://doi.org/10.5216/ree.v20.43943. 


\section{INTRODUÇÃO}

A retinopatia da prematuridade (ROP) é uma doença caracterizada clinicamente pela proliferação anormal de vasos da retina imatura de recém-nascidos prematuros e de baixo peso ao nascer ${ }^{(1)}$. De acordo com a Classificação Internacional da ROP (ICROP), a doença pode ser classificada de acordo com sua gravidade e ordem crescente (estágios 1-5), sua localização (zonas I, II e III), sua extensão em horas (entre uma e doze horas) e a presença de doença plus, uma dilatação arteriolar e tortuosidade venosa, característica indicativa de atividade da doença(2).

A ROP é considerada uma das principais causas de cegueira evitável na infância. Estima-se que anualmente cerca de 400 crianças perdem a visão em decorrência deste agravo. Esse contexto é influenciado pelo nível de cuidados neonatais das instituições por meio de programas de triagem, tratamento e planos. Dentre eles, tem-se o Plano de Ação Mundial para a Prevenção da Cegueira e de Deficiência Visual evitáveis 2014-2019, onde os objetivos se relacionam com a redução da deficiência visual evitável e garantia do acesso aos serviços de reabilitação a todos que dele necessitem ${ }^{(2-3)}$.

A doença é de etiologia multifatorial por estar associada a diversos fatores de riscos que aumentam a probabilidade de sua ocorrência. Porquanto, a unidade de terapia intensiva neonatal (UTIN) como um setor de cuidados críticos e atendimento de pacientes de alta complexidade, torna-se um ambiente vulnerável à ocorrência de erros e eventos adversos ao considerar a susceptibilidade desses pacientes ${ }^{(4-5)}$. Na UTIN, o paciente é manipulado por diversos profissionais, o que predispõe a um aumento da probabilidade de sofrer as consequências de um erro/evento adverso. Por necessitarem de diversas intervenções para diagnóstico e tratamento, o tempo de internamento tende a aumentar, o que também concorre para maior exposição a potenciais $\operatorname{riscos}^{(6)}$.

Neste contexto de vulnerabilidade dos pacientes internados na UTIN, torna-se relevante abordar o Programa Nacional de Segurança do Paciente (PNSP), cujo objetivo principal é a qualificação do cuidado em saúde em todos os estabelecimentos de saúde em território nacional, com prioridade na segurança do paciente no sentido de reduzir erros/eventos adversos ${ }^{(7)}$.

Este estudo torna-se relevante, pois a sumarização dos fatores de risco permitirá a reunião de todos os fatores presentes na literatura que predispõem a ocorrência da ROP. Uma vez conhecendo os fatores, os profissionais de saúde disporão de maiores informações para a detecção precoce da ROP, favorecendo a melhoria da qualidade do cuidado e uma assistência livre de riscos à saúde.

Diante disso, este estudo objetivou sumarizar os fatores de risco para ROP em recém-nascidos internados em UTIN a partir de estudos primários publicados em bases de dados.

\section{MÉTODOS}

Trata de um estudo de revisão integrativa da literatura, caracterizado por um método rigoroso para o estabelecimento de critérios definidos sobre a concepção da questão de pesquisa, amostragem e coleta de dados, análise e apresentação dos resultados a partir de um protocolo de pesquisa previamente elaborado. Tem por finalidade reunir e sintetizar resultados de estudos primários sobre um tema delimitado ou questão de pesquisa. 
Por ser um dos métodos de pesquisa utilizados na prática baseada em evidências, permite a incorporação dessas evidências na conduta clínica de diversas áreas de atenção à saúde ${ }^{(8-11)}$.

Este estudo de revisão foi organizado de acordo com os seguintes passos: identificação do problema de pesquisa e a questão norteadora; busca na literatura mediante aplicação de critérios de inclusão e exclusão; coleta de dados por meio de um instrumento previamente formatado; análise dos dados e apresentação da revisão(9,12).

A questão norteadora para a operacionalização desta revisão foi: Quais os fatores de risco para desenvolvimento de retinopatia da prematuridade em neonatos internados em unidade de terapia intensiva neonatal?

A busca, nas bases de dados, ocorreu nos meses de setembro a dezembro de 2015 mediante uso do proxy licenciado pela Universidade Federal do Rio Grande do Norte por meio da Coordenação de Aperfeiçoamento de Pessoal de Nível Superior (CAPES) (http://www.capes.gov.br/), acessado via Portal de Periódicos CAPES (http://www-periodicos-capes-gov-br.ez18.periodicos.capes.gov.br/) nas seguintes bases de dados: SCOPUS (Elsevier), PubMed Central - PMC, Science Direct (Elsevier), Web of Science - Coleção Principal e Cumulative Index to Nursisng and Allied Heath Literature - CINAHL.

Para busca nas bases, foram utilizados os descritores indexados e suas respectivas sinonímias no Medical Subject Headings (MeSH):

- 1\# ("Premature Birth" OR “Birth, Premature" OR "Births, Premature" OR “Premature Births" OR "Preterm Birth" OR "Birth, Preterm" OR "Births, Preterm" OR "Preterm Births" OR “ Infants, Premature" OR “Premature Infant" OR “Preterm Infants" OR “Infant, Preterm" OR “Infants, Preterm" OR "Preterm Infant" OR “Premature Infants" OR “Neonatal Prematurity" OR “Prematurity, Neonatal”),

- 2\# (“Risk Factors" OR “Factor, Risk" OR “Factors, Risk" OR “Risk Factor”),

- 3\# ("Retinopathy of Prematurity" OR "Prematurity Retinopathies" OR "Prematurity Retinophaty" OR “Retrolental Fibroplasia" OR “Fibroplasia, Retrolental” OR “Fibroplasias, Retrolental” OR "Retrolental Fibroplasias")

- 4\# ("Intensive Care Units", "Neonatal OR Neonatal ICU” OR “Neonatal Intensive Care Units" OR “Newborn Intensive Care Units" OR “Newborn Intensive Care Units (NICU)" OR “ICU, Neonatal" OR "ICUs, Neonatal” OR “Neonatal ICUs" OR “Newborn ICU” OR "ICU, Newborn” OR “ICUs, Newborn” OR “Newborn ICUs”).

Vale ressaltar que as sinonímias foram utilizadas com a finalidade de identificar o maior número possível de publicações relacionadas ao tema em estudo. Os cruzamentos nas bases de dados ocorreram com o uso do operador AND, a saber: 1\# AND 3\# AND 4\# e 2\# AND 3\# AND 4\#.

Os critérios de inclusão foram: artigos completos disponíveis nas bases de dados e artigos que abordaram os fatores de risco para ROP em neonatos internados em UTI. Os editoriais, cartas ao editor, resumos, opinião de especialistas, outras revisões, correspondências, resenhas, capítulos de livros, teses e dissertações foram excluídos da busca. Não foi estabelecido recorte temporal com a finalidade de explorar o máximo possível das publicações sobre o assunto proposto.

A pré-seleção dos estudos ocorreu mediante leitura dos títulos, resumos e aplicação do teste de relevância (composto por critérios de inclusão e exclusão). Artigos repetidos nas bases de dados foram excluídos e em seguida foi realizada a leitura na íntegra dos textos que foram selecionados para a amostra. 
Para a análise e extração dos dados, foi elaborado um instrumento com os seguintes dados: identificação da publicação (título do artigo, bases de dados indexadas, autores, país, idioma e ano de publicação), nome da revista científica, aspectos metodológicos do estudo (método empregado, tipo de abordagem e objetivo ou questão de investigação do estudo), fatores de risco, limitações e conclusões.

Para avaliação crítica dos estudos, identificou-se a classificação do tipo de evidências das pesquisas a partir do referencial do Centro Colaborador do Instituto Joanna Briggs (JBI), que classifica as evidências de acordo com o delineamento metodológico dos estudos (Nível I - Evidência obtida a partir de revisão sistemática de ensaios clínicos controlados randomizados; Nível II - Evidência obtida a partir de ensaio clínico controlado randomizado; Nível III.1 - Evidência obtida de ensaios clínicos controlados bem delineados, sem randomização; Nível III.2 Evidência obtida de estudos de coorte bem delineados ou caso-controle; Nível III.3 - Evidência obtida a partir de séries temporais múltiplas, com ou sem intervenção e resultados dramáticos em experimentos não controlados e Nível IV - Pareceres de autoridades respeitadas, baseados em critérios clínicos e experiência, estudos descritivos ou relatórios de comitês de especialistas) $)^{(13)}$. As categorias surgiram a partir da síntese temática dos dados, estes foram agrupados e nomeados por similaridade dos fatores de risco associados com o desenvolvimento da ROP(10).

A busca resultou em um total de 4.831 artigos. Destes, 26 foram selecionados para compor a amostra final da revisão. A Figura 1 representa a seleção dos estudos. Os resultados foram apresentados de forma descritiva e em tabelas.

Figura 1: Síntese do processo de seleção dos estudos.

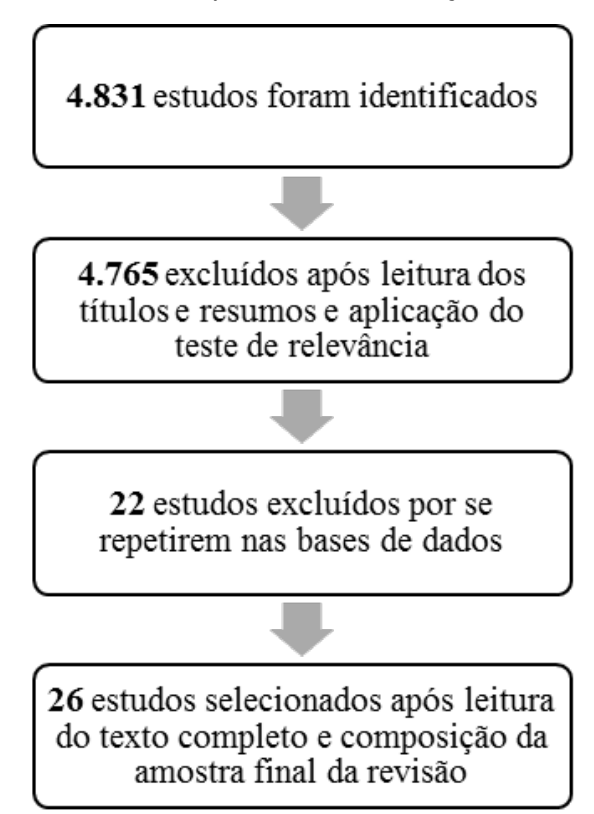

\section{RESULTADOS}

Os estudos foram distribuídos conforme país, ano de publicação, idioma, periódico, método empregado, tipo de abordagem e nível de evidência, conforme demonstrado na Tabela 1. Dentre os países de publicação dos 26 estudos selecionados, os Estados Unidos da América (EUA) se destacaram em 23,1\% dos artigos. Os estudos publicados nos últimos cinco anos somaram um total de $65,4 \%$ da amostra. Todos os artigos foram disponibilizados no idioma inglês. 
Tabela 1: Distribuição dos estudos conforme país, ano, idioma, periódico, metodologia empregada, tipo de abordagem e nível de evidência. Natal, RN, Brasil, 2016.

\begin{tabular}{|c|c|c|}
\hline Variáveis & Frequência & $\%$ \\
\hline \multicolumn{3}{|l|}{ País de publicação } \\
\hline Estados Unidos da América (EUA) & 6 & $23,1 \%$ \\
\hline China & 5 & $19,2 \%$ \\
\hline Irã & 3 & $11,5 \%$ \\
\hline Brasil & 2 & $7,8 \%$ \\
\hline Egito & 2 & $7,8 \%$ \\
\hline Turquia & 2 & $7,8 \%$ \\
\hline Arábia Saudita & 1 & $3,8 \%$ \\
\hline Grécia & 1 & $3,8 \%$ \\
\hline Índia & 1 & $3,8 \%$ \\
\hline Itália & 1 & $3,8 \%$ \\
\hline México & 1 & $3,8 \%$ \\
\hline Romênia & 1 & $3,8 \%$ \\
\hline Total & 26 & $100 \%$ \\
\hline \multicolumn{3}{|l|}{ Ano de publicação } \\
\hline 2011 & 6 & $23,1 \%$ \\
\hline 2012 & 3 & $11,5 \%$ \\
\hline 2014 & 3 & $11,5 \%$ \\
\hline 2015 & 3 & $11,5 \%$ \\
\hline 2007 & 2 & $7,8 \%$ \\
\hline 2010 & 2 & $7,8 \%$ \\
\hline 2013 & 2 & $7,8 \%$ \\
\hline 2002 & 1 & $3,8 \%$ \\
\hline 2004 & 1 & $3,8 \%$ \\
\hline 2006 & 1 & $3,8 \%$ \\
\hline 2008 & 1 & $3,8 \%$ \\
\hline 2009 & 1 & $3,8 \%$ \\
\hline Total & 26 & $100,0 \%$ \\
\hline \multicolumn{3}{|l|}{ Idioma de publicação } \\
\hline Inglês & 26 & $100 \%$ \\
\hline Total & 26 & $100 \%$ \\
\hline \multicolumn{3}{|l|}{ Periódico } \\
\hline Journal of AAPOS & 3 & $11,5 \%$ \\
\hline Brazilian Journal of Ophthalmology & 2 & $7,8 \%$ \\
\hline Introducion Journal of Ophthalmology & 2 & $7,8 \%$ \\
\hline Iran Journal Pediatrics & 2 & $7,8 \%$ \\
\hline The Journal of Maternal-fetal and Neonatal Medicine & 1 & $3,8 \%$ \\
\hline Journal of Perinatology & 1 & $3,8 \%$ \\
\hline Graefes Archives Clinical Exp Ophthalmology & 1 & $3,8 \%$ \\
\hline The Journal of Pediatrics & 1 & $3,8 \%$ \\
\hline Indian Journal Pediatrics & 1 & $3,8 \%$ \\
\hline China Medical Journal & 1 & $3,8 \%$ \\
\hline Journal of Pediatrics Ophthalmology e Strabismus & 1 & $3,8 \%$ \\
\hline Revista de Investigación Clínica & 1 & $3,8 \%$ \\
\hline Clinical Ophthalmology & 1 & $3,8 \%$ \\
\hline American Journal of Ophthalmology & 1 & $3,8 \%$ \\
\hline Early Human Development & 1 & $3,8 \%$ \\
\hline
\end{tabular}


Tabela 1: Distribuição dos estudos conforme país, ano, idioma, periódico, metodologia empregada, tipo de abordagem e nível de evidência. Natal, RN, Brasil, 2016. [continuação]

\begin{tabular}{|c|c|c|}
\hline Variáveis & Frequência & $\%$ \\
\hline Ophthalmology & 1 & $3,8 \%$ \\
\hline São Paulo Journal of Medicine & 1 & $3,8 \%$ \\
\hline Pediatrics International & 1 & $3,8 \%$ \\
\hline Middle East Africann Journal of Ophthalmology & 1 & $3,8 \%$ \\
\hline Pediatrics and Neonatology & 1 & $3,8 \%$ \\
\hline World Journal of Pediatrics & 1 & $3,8 \%$ \\
\hline Total & 26 & $100,0 \%$ \\
\hline \multicolumn{3}{|l|}{ Metodologia empregada } \\
\hline Estudo de coorte & 20 & $76,9 \%$ \\
\hline Estudo de caso-controle & 4 & $15,3 \%$ \\
\hline Estudo transversal & 2 & $7,8 \%$ \\
\hline Total & 26 & $100,0 \%$ \\
\hline \multicolumn{3}{|l|}{ Abordagem } \\
\hline Quantitativa & 26 & $100 \%$ \\
\hline Total & 26 & $100 \%$ \\
\hline \multicolumn{3}{|l|}{ Nível de evidência } \\
\hline III. 2 & 23 & $88,4 \%$ \\
\hline IV & 2 & $7,8 \%$ \\
\hline III.3 & 1 & $3,8 \%$ \\
\hline Total & 26 & $100,0 \%$ \\
\hline
\end{tabular}

A maior frequência de publicações ocorreu no Journal of American Association for Pediatric Ophthalmology and Strabismus (AAPOS) com três estudos (11,5\%), seguido pelo Brazilian Journal of Ophthalmology, Introducion Journal of Ophthalmology e Iran Journal Pediatrics com dois $(7,8 \%)$ estudos cada. A respeito do método empregado, os estudos de coorte predominaram (76,9\%), seguidos pelos estudos de caso-controle $(15,3 \%)$.

Com relação ao tipo de abordagem, todas as pesquisas da amostra utilizaram a quantitativa. Em $23(88,4 \%)$ artigos, a classificação do nível de evidência foi III.2, em dois (7,8\%) foi IV e em um $(3,8 \%)$ foi III.3.

Após análise, foi realizada a síntese temática dos dados e os resultados foram organizados em categorias para caracterização dos fatores de risco para ROP no ambiente de terapia intensiva neonatal. Os fatores de risco em forma de categorias foram classificados pelo número de estudos que os abordaram, conforme demonstra a Tabela 2. 
Tabela 2: Categorização dos fatores de risco para retinopatia da prematuridade em unidade de terapia intensiva neonatal $(n=26)$. Natal, RN, Brasil, 2016.

\begin{tabular}{|c|c|c|}
\hline Fatores de risco & $\mathbf{N}$ & $\%$ \\
\hline \multicolumn{3}{|l|}{ Relacionados à terapêutica clínica } \\
\hline Terapia com oxigênio ${ }^{(14-17,18-22)}$ & 9 & $34,6 \%$ \\
\hline Ventilação mecânica ${ }^{(16-17,19-21,23-25)}$ & 8 & $30,7 \%$ \\
\hline Transfusão de sangue ${ }^{(15-16,19,21-22,24,26)}$ & 7 & $26,9 \%$ \\
\hline Alta permanência na UTIN ${ }^{(17,23)}$ & 2 & $7,6 \%$ \\
\hline \multicolumn{3}{|l|}{ Relacionados ao uso de medicamentos } \\
\hline Uso de eritropoietina humana recombinante ${ }^{(21,27)}$ & 2 & $7,6 \%$ \\
\hline Uso de esteroides após o nascimento ${ }^{(25)}$ & 1 & $3,8 \%$ \\
\hline Inalação de óxido nítrico ${ }^{(23)}$ & 1 & $3,8 \%$ \\
\hline Uso de surfactante $\mathrm{e}^{(19)}$ & 1 & $3,8 \%$ \\
\hline \multicolumn{3}{|l|}{ Comorbidades relacionadas à ROP* } \\
\hline Função pulmonar comprometida ${ }^{(19,24,28-29)}$ & 4 & $15,3 \%$ \\
\hline Hemorragia intraventricular ${ }^{(24-25,30)}$ & 3 & $11,5 \%$ \\
\hline Anemia $^{(22,31)}$ & 2 & $7,6 \%$ \\
\hline Candidemia ${ }^{(32)}$ & 1 & $3,8 \%$ \\
\hline \multicolumn{3}{|l|}{ Características neonatais } \\
\hline BPN**(14-15,18-26,28-31,33-35) & 17 & $65,3 \%$ \\
\hline Baixa IG***(14-15,17-19,21-25,28,30,31,33-36) & 17 & $65,3 \%$ \\
\hline Sepse $e^{(15,16,19,24-25)}$ & 5 & $19,2 \%$ \\
\hline PCA $* * * *(19,24-25)$ & 3 & $11,5 \%$ \\
\hline Raça não negra ${ }^{(37)}$ & 1 & $3,8 \%$ \\
\hline Sexo masculino ${ }^{(37)}$ & 1 & $3,8 \%$ \\
\hline \multicolumn{3}{|l|}{ Características clínicas associadas à ROP* } \\
\hline Hiperglicemia $1(17,24,38-39)$ & 4 & $15,3 \%$ \\
\hline Hipotensão ${ }^{(17,19,25)}$ & 3 & $11,5 \%$ \\
\hline Apneia $^{(17,19,31)}$ & 3 & $11,5 \%$ \\
\hline Hipóxia pós-natal(22) & 1 & $3,8 \%$ \\
\hline Baixo Índice de Apgar ${ }^{(25)}$ & 1 & $3,8 \%$ \\
\hline \multicolumn{3}{|l|}{ Fatores maternos } \\
\hline Pré-eclâmpsia materna $(14,17,25)$ & 3 & $11,5 \%$ \\
\hline Descolamento de placenta(31) & 1 & $3,8 \%$ \\
\hline Múltiplas gestações ${ }^{(17)}$ & 1 & $3,8 \%$ \\
\hline
\end{tabular}

\section{DISCUSSÃO}

Foi observado que a maioria dos artigos que abordou os fatores de risco para ROP na UTIN foi publicada nos últimos cinco anos. Isso demonstra a relevância da discussão na atualidade. No entanto, a classificação do nível de evidência aponta a necessidade de ampliação e aprofundamento das pesquisas na área, no sentido de promover uma prática baseada em alto grau de evidência.

Destaca-se que todos os estudos que abordaram os fatores de risco para a ROP em UTIN que foram incluídos nesta revisão fundamentaram a síntese temática dos dados. Desta forma, a discussão será baseada nas categorias estabelecidas. 


\section{Relacionados à terapêutica clínica}

A categoria terapia com oxigênio foi discutida em $34,6 \%{ }^{(14-22)}$ dos estudos. $O$ efeito do oxigênio, na retina de recém-nascido prematuro, ocorre em duas fases, a saber: a fase vasoconstritora e a fase vasoproliferativa. $\mathrm{Na}$ primeira, a hiperóxia retinal desencadeia a supressão da proliferação normal dos vasos e a vasoconstrição. Enquanto que na fase vasoproliferativa, quando o oxigênio se desloca para o ar, os níveis do fator de crescimento endotelial dos vasos aumentam e provocam uma vasoproliferação anormal.(40) 0 número de dias de oxigenoterapia também esteve significativamente associado ao desenvolvimento de qualquer fase de ROP(41). Estudo $^{(42)}$ retrospectivo realizado na Inglaterra, que examinou a incidência da doença e a política de suplementação de oxigênio em cinco UTINs, mostrou que os bebês nascidos antes de 28 semanas de gestação e que utilizaram oxigênio suplementar desenvolveram ROP.

A ventilação mecânica esteve presente em $30,7 \%$ dos artigos ${ }^{(16-17,19-21,23-25)}$ selecionados. Sua associação com o desenvolvimento de ROP ainda não é clara e um estudo destacou que essa falta de associação pode ter sido devido ao uso limitado da suplementação do oxigênio nos prematuros durante o período do estudo ${ }^{(43)}$. Ressalta ainda que o real problema da ventilação mecânica para o desenvolvimento da ROP é a exposição intensiva a níveis de oxigênio sob alta pressão ${ }^{(43)}$. Em contrapartida, outro estudo ${ }^{(15)}$ não encontrou associação significativa entre a ocorrência de ROP e o tempo da ventilação mecânica.

Sabe-se que a maioria dos neonatos em UTIN necessita de algum tipo de intervenção com o oxigênio devido à imaturidade pulmonar. Diante disso, indica-se investigar mais detalhadamente se o fator de risco para ROP é o oxigênio ou a condição que leva ao seu uso.

A transfusão de sangue, como fator relacionado à terapia, representou $26,9 \%^{(15-16,19,21-22,24,26)}$ dos artigos da amostra. Um estudo ${ }^{(44)}$ revelou que o volume das transfusões é um fator independente para o aumento da incidência da ROP. A transfusão de sangue causa um aumento nos níveis do fator de crescimento semelhante à insulina, este fator acaba por estimular uma neovascularização da retina e desenvolver a ROP. Além disso, as transfusões repetidas com hemoglobina adulta pode provocar hiperoxemia devido sua baixa afinidade pelo oxigênio e causar dano oxidativo nos vasos retinianos ${ }^{(44-46)}$.

Com relação à longa permanência na UTIN ${ }^{(17,23)}$, apesar da escassez de estudos que demonstrassem a relação desse fator com a ROP, um dos estudos ${ }^{(23)}$ selecionados mostrou que os dias de estadia na UTIN tiveram uma associação significativa com a ocorrência da retinopatia, principalmente quando a permanência é maior que 28 dias $^{(17)}$.

\section{Relacionados ao uso de medicamentos}

O uso de eritropoietina humana recombinante foi encontrado em 7,6\% da amostra ${ }^{(21,27)}$. Esse medicamento é administrado em prematuros com o objetivo de ser uma agente neuroprotetor, com vistas ao comprometimento neurológico desses recém-nascidos ${ }^{(47)}$. Alguns estudos demonstraram que a associação entre o uso da eritropoietina e a ROP grave pode ser induzida pelo oxigênio ${ }^{(48-49)}$. Em um modelo de ratos transgênicos, observouse que a eritropoietina promoveu uma angiogênese intravítrea, o que poderia levar ao desenvolvimento da $\mathrm{ROP}^{(50)}$. 
O uso de esteroides após o nascimento, foi encontrado em 3,8\% da amostra ${ }^{(25)}$. É comum em prematuros, pois ajuda no amadurecimento dos pulmões, porém seu uso deve ser feito com cautela ${ }^{(43)}$. A literatura é escassa em relação aos mecanismos desencadeados pelo uso dos esteroides no desenvolvimento da ROP, no entanto os estudos revelaram que seu uso está relacionada com patologia(25,43).

A inalação de óxido nítrico (iNO) foi descrita em um estudo ${ }^{(23)}$ como um novo fator de risco para a ROP. A relação entre a iNO e a ROP deve-se ao fato de que o óxido nítrico aumenta a saturação de oxigênio e causa hiperóxia. Isso causa danos aos vasos da retina e leva à uma vascularização incompleta da retina prematura(51). Embora seja elencado como fator de risco para o desenvolvimento da ROP, o tratamento com o iNO é uma nova modalidade de escolha para recém-nascidos com hipertensão pulmonar que não respondem à ventilação mecânica, para reduzir a insuficiência respiratória, pois causa vasodilatação pulmonar e por consequência melhora a oxigenação(41,52).

O uso de surfactante foi abordado em $3,8 \%{ }^{(19)}$ dos estudos da amostra final. O surfactante exógeno é usado em recém-nascidos com angústia respiratória, pois aumenta os níveis de oxigênio corporal e sua relação com a ROP é devido a isso, já que o oxigênio tem relação com a patogênese da ROP(52). Um estudo retrospectivo ${ }^{(41)}$ revelou que a terapia com surfactante em prematuros está associada com a ROP.

O uso de medicamentos, como a eritropoietina, esteroides, óxido nítrico e surfactante também estão relacionados à avaliação e conduta do enfermeiro aplicado a manuseio e controle rigoroso da administração destes medicamentos, tendo em vista a alta prevalência destes procedimentos em neonatos e por ser diretamente proporcional a presença de retinopatia na prematuridade.

\section{Comorbidades relacionadas à ROP}

A função pulmonar comprometida foi discutida em $15,3 \%$ dos estudos ${ }^{(19,24,28-29)}$. Esta categoria incluiu a síndrome do desconforto respiratório (SDR) e outras doenças crônicas pulmonares. Recém-nascidos com a SDR apresentam variações na saturação de oxigênio e essa instabilidade pode predispor à ROP(49).

A hemorragia intraventricular esteve presente em três estudos ${ }^{(24-25,30)}$. Estes revelaram que a ROP pode ocorrer em conjunto com essa comorbidade. Com relação à categoria anemia, presente em $7,6 \%$ dos estudos ${ }^{(22,31)}$, um deles ${ }^{(31)}$ realizado com 639 recém-nascidos mostrou que 10,8\% desenvolveram a ROP e a anemia foi considerada significativamente associada.

A categoria candidemia foi abordada em 3,8\% da amostra como fator de risco para a ROP. Um estudo(32) definiu a candidemia como isolamento de espécies de Cândida em pelo menos uma hemocultura. Esse achado corrobora com outro trabalho ${ }^{(53)}$ que encontrou uma relação de aumento da incidência de ROP limiar em crianças com Cândida. A suposta relação entre a candidíase e a ROP presume que a infecção pode estimular a produção de citocinas e fatores angiogênicos a partir da retina ${ }^{(32)}$. No entanto, existem poucos estudos atuais sobre o tema que descrevam a relação causal entre a candidemia o desenvolvimento da ROP.

O levantamento de comorbidades relacionadas à ROP evidencia a importância da assistência pré-natal completa como importante componente preventivo para o desenvolvimento da retinopatia da prematuridade, além de ressaltar o papel do enfermeiro no âmbito do cuidado à saúde da gestante, e consequentemente, do neonato. 


\section{Características neonatais}

Dentre os fatores neonatais, o BPN foi encontrado em $65,3 \%$ dos estudos ${ }^{(14-15,18-26,28-31,33-35)}$, que relacionaram o BPN como significativamente associado com o desenvolvimento de ROP, dentre os principais fatores de risco para a doença. Um estudo realizado no Hospital das Clínicas da Universidade Federal de Uberlândia, no sudeste brasileiro, concluiu que o desenvolvimento de ROP foi inversamente proporcional ao $\mathrm{PN}^{(54)}$. Salienta-se que existem variações do peso médio de nascimento para ser considerado como fator de risco entre os estudos. Em um estudo retrospectivo realizado em Cuba, a maior frequência de indivíduos com ROP foi naqueles com peso ao nascer entre 1351 e 1700 gramas $^{(43)}$. Atualmente, o baixo ganho de peso em prematuros que persiste por seis semanas após o nascimento tem sido aceito como fator de risco para o desenvolvimento da $\mathrm{ROP}^{(40)}$.

A baixa idade gestacional foi a segunda categoria mais representativa entre os estudos ${ }^{(14-15,17-19,21-25,28,30-}$ 31,33-36). Destes, 65,3\% demonstraram que a IG inferior a 30 semanas constitui fator de risco para ROP. Quanto menor a idade gestacional, maior é a imaturidade da retina e menor o desenvolvimento vascular ${ }^{(55)}$. Em consonância, outro estudo relatou que a IG $<28$ semanas foi associada com $100 \%$ dos casos de ROP nos prematuros da amostra ${ }^{(54)}$.

Ainda em relação à esses fatores, a sepse esteve presente em $19,2 \%$ estudos $^{(15-16,19,24-25)}$ selecionados. A presença de infecção esteve associada à ROP severa, possivelmente devido à inflamação sistêmica que age sinergicamente com a hiperóxia ${ }^{(56-58)}$. Contudo, um estudo retrospectivo ${ }^{(30)}$ que objetivou determinar a incidência e os fatores de risco para ROP não identificou relação significativa entre sepse e ocorrência da ROP.

No que diz respeito à categoria persistência do canal arterial (PCA), $11,5 \%$ dos estudos ${ }^{(19,24-25)}$ abordam essa condição como fator de risco potencial para ocorrência da ROP. Ressalta-se o número reduzido de estudos que expliquem claramente a relação entre esse fator e a retinopatia, mas acredita-se que a associação se deve às características clínicas da PCA como: perfusão retiniana alterada e anormalidades no fluxo sanguíneo para a retina $^{(59)}$.

A raça não negra e o sexo masculino foram variáveis discutidas em $3,8 \%$ dos estudos ${ }^{(37)}$. Essa mesma pesquisa ${ }^{(37)}$ encontrou uma ocorrência aumentada de ROP em indivíduos de raças que não fossem a negra, porém trata de uma evidência moderada, de acordo com o tipo do estudo. O mesmo estudo evidenciou que recémnascidos do sexo masculino apresentaram uma incidência maior de ROP, no entanto, sua associação com a ROP não foi clara e novos estudos devem ser realizados nesse sentido.

\section{Características clínicas associadas à ROP}

A variável hiperglicemia foi discutida por $15,3 \%$ dos artigos ${ }^{(17,24,38-39)}$. As concentrações elevadas de glicose no sangue de recém-nascidos também aumentam o risco para desenvolver ROP, tanto as formas mais leves como as graves, pois os níveis altos do fator de crescimento semelhante à insulina tipo 1 (IGF-1) elevam os níveis do fator de crescimento endotelial, principal determinante da ROP(41).

A hipotensão neonatal ${ }^{(17,19,25)}$ foi descrita em $11,5 \%$ da amostra. Acredita-se que sua associação com a ROP seja devido à queda nos níveis de oxigênio que afetam os vasos retinianos de prematuros ${ }^{(25)}$. 
A apneia foi discutida em $11,5 \%$ dos artigos ${ }^{(17,19,31)}$ selecionados. Apesar de outros estudos não retratarem este fator como de risco para a ROP, foi identificado nas pesquisas selecionadas que a apneia representa um fator de risco significativo para o desenvolvimento da ROP, principalmente quando esta suspensão da respiração tornase maior que 20 segundos $^{(19,31)}$. A hipóxia pós-natal foi uma categoria que esteve presente em apenas $3,8 \%$ da amostra $^{(22)}$. Porquanto, ressalta-se que sua discussão na literatura ainda é escassa.

O índice de Apgar é utilizado no pós-parto para avaliar a adaptação do recém-nascido à vida extrauterina. Além disso, é importante para avaliar as condições fisiológicas e para identificar a necessidade de reanimação ou outro cuidado especial ${ }^{(60)}$. Com base nisso, 3,8\% dos estudos ${ }^{(25)}$ selecionados referiram a categoria baixo Índice de Apgar como fator de risco associado com a ROP. Outras pesquisas ${ }^{(41,61)}$ identificaram que os baixos valores de Apgar entre o 1으 e 5o minutos de vida estão significativamente associados com a ROP, haja vista que bebês prematuros tendem a apresentar baixos valores no Apgar.

Além disso, existe um escore que avalia o risco clínico do neonato, o Índice de Risco Clínico para Bebês, calculado a partir de seis variáveis medidas durante as primeiras 12 horas após o nascimento: peso ao nascer, idade gestacional, malformação congênita, excesso de base e a fração inspiratória de oxigênio (FiO2) adequada. Quanto maior esse índice, maior o risco de desenvolvimento da ROP(32).

\section{Fatores maternos}

Esses fatores ainda são pouco discutidos na literatura e a associação deles com a ROP ainda não é bem clara, seria importante que novos estudos fossem propostos nesse sentido. A pré-eclâmpsia materna foi abordada por $11,5 \%$ da amostra ${ }^{(14,17,25)}$. A pré-eclâmpsia é caracterizada, em nível vascular, por um conjunto de alterações na vasculatura materno-fetal, como alterações isquêmicas, enfartes e até mesmo o descolamento da placenta ${ }^{(62)}$. Tais condições podem levar à um suprimento inadequado do oxigênio para o feto e causar danos vasculares na retina ${ }^{(14)}$. Um dos estudos relatou a pré-eclâmpsia materna como um fator de proteção significativo para a ROP(30).

A categoria descolamento de placenta esteve presente em um estudo ${ }^{(31)}$, o que reforça a necessidade de novas pesquisas na área para esclarecer melhor o papel e a relação dessa condição com a ROP. Um estudo(31) avaliou os fatores de risco maternos e neonatais para a ROP e evidenciou o descolamento de placenta com fator materno que tem influência independente na ocorrência da ROP.

No que diz respeito à categoria múltiplas gestações, apenas $3,8 \%$ dos estudos ${ }^{(17)}$ abordou essa variável como fator de risco para ocorrência da ROP. Sua associação com a ROP não está esclarecida.

\section{CONCLUSÃO}

Esta revisão contribuiu com o conhecimento sobre os principais fatores de risco para o desenvolvimento da ROP nas unidades de cuidados intensivos neonatais. Por meio deste estudo, verificaram-se duas categorias de fatores de risco subjacente a todas as demais apresentadas: fatores de risco relacionados à terapêutica clínica e características neonatais. Tal fundamento põe em questão o monitoramento da oxigenoterapia, do uso da ventilação mecânica e de transfusões sanguíneas como medidas preventivas realizadas diretamente pela enfermagem. 
Além do mais, ao identificar fatores de risco não modificáveis relacionados principalmente ao baixo peso ao nascer e a baixa idade gestacional, a enfermagem pode contribuir de forma significativa no sentido de garantir uma redução dos demais fatores de risco associados à assistência em saúde e permitir uma triagem oftalmológica que impeça o desenvolvimento da doença, suas sequelas e/ou viabilize tratamento na fase inicial da doença.

Para tanto, o levantamento de comorbidades e características neonatais evidencia a importância da assistência pré-natal completa como importante componente preventivo para o desenvolvimento da retinopatia da prematuridade, além de ressaltar o papel do enfermeiro no âmbito do cuidado à saúde do recém-nascido.

Ainda relacionado a isso, o uso de medicamentos, como a eritropoietina, esteroides, óxido nítrico e surfactante também estão relacionados à avaliação e conduta do enfermeiro aplicado ao manuseio e controle rigoroso da administração destes medicamentos, tendo em vista que a alta frequência destes procedimentos em neonatos pode ser diretamente proporcional à presença de retinopatia na prematuridade.

Além disso, igualmente pertinente, verificou-se a necessidade de ampliação do leque de estudos relacionados aos fatores maternos, cujos resultados evidenciaram gaps na produção do conhecimento em relação à atenção à saúde da gestante voltada para medidas preventivas e diagnosticadoras da retinopatia da prematuridade. Neste sentido, acredita-se que por meio de uma prática baseada em evidências, tais medidas possam futuramente ser aplicadas e contribuírem para redução desta doença.

Ademais, a maioria dos estudos utilizados nesta revisão corresponde aos artigos publicados nos últimos seis anos, o que evidencia que o tema é atual. No entanto, ressalta-se a importância de realização de outras pesquisas com outros delineamentos que representem níveis de evidência mais altos, de forma a confirmar a importância de conhecer e atuar sobre os fatores de risco da ROP como uma forma de prevenção de agravos.

O conhecimento sobre os fatores de risco é importante no sentido de adoção de medidas preventivas, que impeçam o desenvolvimento da ROP e suas possíveis sequelas, bem como a qualificação do cuidado em saúde neonatal e uma assistência livre de riscos à saúde. Esse conhecimento contribui na detecção precoce da retinopatia bem como seu tratamento ainda na fase inicial da doença. Portanto, este estudo torna-se pertinente por sumarizar essas informações e dispor em forma de categorias, fatores de risco que podem ser identificados, previstos e controlados. Uma vez munidos destas informações, profissionais de saúde poderão agir de forma a evitar e até minimizar a ocorrência do fenômeno.

Desta forma, o objetivo do artigo foi alcançado por ter se tratado de uma vasta busca e seleção da literatura pertinente, que possibilitou a reunião dos fatores de risco disponíveis na literatura.

\section{REFERÊNCIAS}

1. Asano MK, Dray PB. Retinopathy of prematurity. Disease-a-Month [Internet]. 2014 [acesso em: 16 abr. 2018];60(6):282-91. Disponível em: http://dx.doi.org/10.1016/j.disamonth.2014.03.009.

2. Conselho Brasileiro de Oftalmologia. Sociedade Brasileira de Pediatira. Retinopatia da prematuridade. Brasil; 2011.

3. Fors MS, Armas MM, Martínez RR, Hernández ML, González YT. Características clínicas epidemiológicas de la retinopatía de la prematuridad en recién nacidos de embarazos múltiples. Revista Cubana de Oftalmología [Internet]. 2013 [acesso em 14 jan 2016];26(1):121-8. Disponível em: http://www.revoftalmologia.sld.cu/index.php/oftalmologia/article/view/174/html.

4. Minuzzi AP, Salum NC, Locks MOH, Amante LN, Matos E. Contributions of healthcare staff to promote patient safety in intensive care. Esc Anna Nery [Internet]. 2016 [acesso em: 16 abr. 2018];20(1):121-9. Disponível em: http://dx.doi.org/10.5935/1414$\underline{8145.20160017 .}$ 
5. Oliveira COP, Souza NL, Silva EMM, Silva JB, Saraiva EM, Rangel CT. Caracterização das infecções relacionadas à assistência à saúde em uma unidade de terapia intensiva neonatal. Rev. enferm. UERJ [Internet]. 2013 [acesso em: 16 abr. 2018];21(1):90-4. Disponível em: http://www.e-publicacoes.uerj.br/index.php/enfermagemuerj/article/view/6370.

6. Sousa DS, Sousa Júnior AS, Santos ADR, Melo EV, Lima SO, Almeida-Santos MA, et al. Morbidity in extreme low birth weight newborns hospitalized in a high risk public maternity. Rev. Bras. Saude Mater. Infant. [Internet]. 2017 [acesso em: 16 abr. 2018];17(1):139-47. Disponível em: http://dx.doi.org/10.1590/1806-93042017000100008.

7. Brasil. Ministério da Saúde. Portaria no 529, de 10 de abril de 2013. Programa Nacional de Segurança do Paciente. Brasília: Ministério da Saúde; 2013.

8. Crossetti MGO. Revisão integrativa de pesquisa na enfermagem o rigor cientifico que lhe é exigido. Rev. Gaúcha Enferm. [Internet]. 2012 [acesso em: 16 abr. 2018];33(2):8-9. Disponível em: http://dx.doi.org/10.1590/S1983-14472012000200001. 9. Teixeira E, Medeiros HP, Nascimento MHM, Silva BAC, Rodrigues C. Revisão Integrativa da Literatura passo-a-passo \& convergências com outros métodos de revisão. Rev Enferm UFPI [Internet]. 2013 [acesso em: 16 abr. 2018];2(spe):3-7. Disponível em: https://doi.org/10.26694/reufpi.v2i5.1457.

10. Soares CB, Hoga LAK, Peduzzi M, Sangaleti C, Yonekura T, Silva DRAD. Revisão integrativa: conceitos e métodos utilizados na enfermagem. Rev Esc Enferm USP [Internet]. 2014 [acesso em 14 mar 2016]; 48(2):335-345. Disponível em:

http://dx.doi.org/10.1590/S0080-6234201400002000020.

11. Pedrosa KKA, Oliveira ICM, Feijão AR, Machado RC. Enfermagem baseada em evidência: caracterização dos estudos no Brasil. Cogitar Enferm[Internet]. 2015[acesso em 04 mai 2016]; 20(4):733-741. Disponível em: http://dx.doi.org/10.5380/ce.v20i4.40768. 12. Souza MT, Silva MD, Carvalho R. Revisão integrativa: o que é e como fazer. Einstein [Internet]. 2010[acesso em 04 mai 2016]; 8(1 Pt 1):102-6. Disponível em: http://www.scielo.br/scielo.php?pid=S1679-45082010000100102\&script=sci arttext\&tlng=pt. 13. Karino ME, Felli VEA. Enfermagem baseada em evidências: avanços e inovações em revisões sistemáticas. Cienc Cuid Saúde [Internet]. 2012[acesso em 06 mai 2016];1(supl):11-5. Disponível em:

http://periodicos.uem.br/ojs/index.php/CiencCuidSaude/article/view/17048/pdf.

14. Chen Y, Xun D, Wang Y-C, Wang B, Geng S-H, Chen H, et al. Incidence and risk factors of retinopathy of prematurity in two neonatal intensive care units in North and South China. Chinese Medical Journal [Internet]. 2015[acesso em 06 set 2015];123(7);914-18. Disponível em: https://www.ncbi.nlm.nih.gov/pmc/articles/PMC4834008/pdf/CMJ-128-914.pdf.

15. Hakeem AHAA, Nohamed GB, Othemam MF. Retinopathy of prematurity: a study of prevalence and risk factors. Middle East Afr J Ophthalmol[Internet]. 2012[acesso em 06 dez 2015];19(3):289-94. Disponível em:

https://www.ncbi.n/m.nih.gov/pmc/articles/PMC3401797/.

16. Giannantonio C, Papacci P, Cota F, Vento G, Tesfagabir MG, Purcaro V, et al. Analysis of risk factors for progression to treatment - requiring retinopathy of prematurity in a single neonatal intensive care unit: is the exposure time relevant?. J Matern Fetal Neonatal Med [Internet]. 2012[acesso em 06 dez 2015];25(5):471-7. Disponível em:

http://www.tandfonline.com/doi/full/10.3109/14767058.2011.587056?scroll=top\&needAccess=true.

17. Martínez-Cruz CF, Salgdo-Valladares M, Poblano A, Trinindad-Pérez MC. Risk factors associated with retinopathy of prematurity and visual alterations in infants with extremely low birth weight. Rev Invest Clin[Internet]. 2012[acesso em 08 set 2015];64(2):13643. Disponivel em: https://pdfs.semanticscholar.org/da21/4ea3a5c07ac6758776e44d41110f3e239f6f.pdf.

18. Ghaseminejad A, Niknafs P. Distribution of retinopathy of prematurity its risk factors. Iran J Pediatr[Internet]. 2011[acesso em 15 set 2015];21(2):209-14. Disponível em: https://www.ncbi.nlm.nih.gov/pmc/articles/PMC3446155/pdf/IJPD-21-209.pdf.

19. Kumar P, Sankar MJ, Deorari A, Azad R, Chandra P, Agarwol R, et al. Risk factors for severe retinopathy of prematurity in preterm low birth weight neonates. Indian J Pediatr [Internet].2011[acesso em 15 nov 2015];78(7):812-6. Disponível em: https://link.springer.com/article/10.1007\%2Fs12098-011-0363-7.

20. Makhtari MB, Pishva N, Attarzadeh A, Hosseini H, Pourarian S. Incidence and risk factors of retinopathy of prematurity among preterm infants in Shiraz/Iran. Iran J Pediatr [Internet]. 2010[acesso em 15 nov 2015];20(3):303-7. Disponível em:

http://www.bioline.org.br/pdf?pe10045.

21. Filho JBF, Eckert GU, Valiatti FB, Santos PGB, Costa MC, Pracianoy RS. The influence of gestational age on the dynamic behavior of other risk factors associated with retinopathy of prematurity. Graefes Arch Clin Exp Ophthalmol [Internet]. 2010 [acesso em 24 set 2015];248:893-900. Disponível em: https://www.ncbi.nlm.nih.gov/pmc/articles/PMC2859157/.

22. Liu L, Tian T, Zheng C-X, lleana V, Ioana A, Tatiana C. Risk factors and laser-therapy for retinopathy of prematurity in neonatal intensive care unit. World J Pediatr [Internet]. 2009[acesso em 04 out 2015];5(4):304-7. Disponível em:

https://link.springer.com/article/10.1007\%2Fs12519-009-0058-6.

23. Sorge AJ, Termate JUM, Kerkhaff FT, Rijn L, Simonsz HJ, Peer PGM. Nationwide inventory of risk factors for retinopathy of prematurity in the Netherlands. J Pediatr[Internet]. 2014[acesso em 04 out 2015];164:494-8. Disponível em:

http://www.jpeds.com/article/S0022-3476(13)01424-8/fulltext.

24. Abdel AM, Hadi, Hamdy IS. Correlation between risk factors during the neonatal period and appearance of retinopathy of prematurity in preterm infants in neonatal intensive care units in Alexandria, Egypt. Clinical Ophthalmology [Internet]. 2013[acesso em 15 nov 2015];7:831-7. Disponível em: https://www.ncbi.nlm.nih.gov/pmc/articles/PMC3652516/. 
25. Yang C-YY, Lin R, Yang P-H, Chu S-M, Hsu J-F, Fu R-H, et al. Analysis of incidence and risk factors of retinopathy of prematurity among very-low-birth-weight infants in North Taiwan. Pediatrics and neonatology [Internet]. 2011[acesso em 15 dez 2015];52:3216. Disponível em: https://linkinghub.elsevier.com/retrieve/pii/S1875-9572(11)00120-3.

26. Mehmet S, Fusun A, Sebnem C, Ozgur O, Gultan E, Taylon AO, et al. One-year experience in the retinopathy of prematurity: frequency and risk factors, short-term results and fallow-up. Int J Ophthalmol [Internet]. 2011[acesso em 15 out 2015];4(6):634-40. Disponível em: https://www.ncbi.nlm.nih.gov/pmc/articles/PMC3340803/pdf/ijo-04-06-634.pdf.

27. Suk KK, Dunbar JA, Liw A, Daher NS, Leng CKL, Leng JK, et al. Huaman recombinant erythropoietin and the incidence of retinopathy of prematurity: a multiple regression model. Journal of AAPOS[Internet]. 2008[acesso em 23 out 2015];12(3):233-8. Disponível em: https://linkinghub.elsevier.com/retrieve/pii/S1091-8531(07)00449-1.

28. Giapros V, Drougia A, Asproudis I, Theocharis P, Andronikou S. Low gestational age and chronic lung disease are synergistic risk factors for retinopathy of prematurity. Early Human Development [Internet]. 2011[acesso em 23 out 2015];87:653-7. Disponível em: http://www.sciencedirect.com/science/article/pii/S0378378211001861?via\%3Dihub.

29. Akkoyun J, Oto S, Yilmaz G, Gurakan B, Tarcan A, Anuk D, Akgun S, et al. Risk factors in the development of mild and severe retinopathy of prematurity. Journal of AAPOS [Internet]. 2006[acesso em 23 out 2015];10(5):449-53. Disponível em:

http://www.jaapos.org/article/S1091-8531(06)00447-2/fulltext.

30. Yau GSK, Lu JWY, Tam VTY, Liu CCL, Chu BCV, Yun CYF. Incidence and risk factors for retinopathy of prematurity in extreme low birth weight chinese infants. Int Ophthalmol [Internet]. 2015[acesso em 13 dez 2015];35:365-73. Disponível em:

https://www.ncbi.nlm.nih.gov/pubmed/24898774.

31. Chen Y, Li X-X, Yin H, Gibert C, Liang J-h, Jiang Y-r, et al. Risk factors for retinopathy of prematurity in six neonatal intensive care units in Bejing, China. Br J Ophthalmol [Internet]. 2008[acesso em 20 set 2015];92:326-31. Disponível em:

http://bjo.bmj.com/content/92/3/326.long.

32. Noyola DE, Bohra L, Paysse EA, Fernandez M, Coats DK. Association of candidemia and retinopathy of prematurity in very low birth weight infants. Ophthalmology [Internet]. 2002[acesso em 11 set 2015];109(1):80-4. Disponível em:

https://linkinghub.elsevier.com/retrieve/pii/S0161-6420(01)00841-7.

33. Pierce LM, Raab EL, Holzman IR, Ginsburg RN, Brodil SE, Stroustrup A. Importance of birth weight as a risk fator for severe retinopathy of prematurity when gestational age is 30 or more weeks. Am J Ophthalmol [Internet]. 2014[acesso em $13 \mathrm{dez}$ 2015];157:1227-30. Disponível em: https://www.ncbi.nlm.nih.gov/pmc/articles/PMC4430094/.

34. Isaza G, Arora S, Bal M, Chaudhary V. Incidence of retinopathy of prematurity and risk factors among premature infants at a neonatal intensive care unit in Canada. J Pediatr Ophthalmol Strabismus [Internet]. 2013[acesso em 17 dez 2015];58:27-32. Disponível em: https://www.healio.com/ophthalmology/journals/jpos/2013-1-50-1/\%7B1a5f873c-3d0a-45cd-aab7-

37eabf12cdd9\%7D/incidence-of-retinopathy-of-prematurity-and-risk-factors-among-premature-infants-at-a-neonatal-intensivecare-unit-in-canada.

35. Binkhathlan AA, Almahmoud LA, Saleh MJ, Srugeri S. Retinopathy of prematurity in Saudi Arabia: incidence, risk factors, and the applicability of current screening criteria. Br J Ophathalmol [Internet]. 2008[acesso em 19 out 2015];92:167-9. Disponível em: http://bjo.bmj.com/content/92/2/167.long.

36. Gonçalves E, Násser LS, Martelli DR, Alkimim IR, Mourão TV, Caldeira AP, et al. Incidence and risk factors for retinopathy of prematurity in a brazilian reference servisse. São Paulo Med J [Internet]. 2014[acesso em 15 dez 2015];132(2):85-91. Disponível em: http://www.scielo.br/pdf/spmj/v132n2/1516-3180-spmj-132-02-00085.pdf.

37. Yang MB, Donovan EF, Wagge JR. Race, gender, and Clinical Risk Index for Babies (CRIB) Score as preditors of severe retinipathy of prematurity. Journal of AAPOS [Internet\}. 2006[acesso em 15 dez 2015];10(3):253-61. Disponível em:

http://www.jaapos.org/article/S1091-8531(06)00006-1/fulltext.

38. Ahmadpour-Kacho M, Motlagh J, Rasoulinejad SA, Jahamgir T, Bijni A, Pasha YZ. Correlation between hyperglycemia and retinopathy of prematurity. Pediatrics International [Internet]. 2014[acesso em 05 out 2015];56:726-30. Disponível em: https://www.ncbi.nlm.nih.gov/pubmed/24803073.

39. Kaempf JW, Kaempf AJ, Wu J, Stawarz M, Niemeyer J, Grunkemieier G. Hyperglycemia, insulin and slower growth velocity may increase the risk for retinopathy of prematurity. Journal of Perninatology [Internet]. 2011[acesso em 15 dez 2015];31:251-7.

Disponível em: https://www.nature.com/articles/ip2010152.

40. Shah PK, Prabhu V, Karandikar SS, Ranjan R, Naredran V, Kalpana N. Retinopathy of prematurity: past, presente and future. World J. Clin Pediatr [Internet]. 2016[acesso em 19 jul 2016];5(1):35-46. Disponível em:

https://www.ncbi.nlm.nih.gov/pmc/articles/PMC4737691/.

41. Slidsborg C, Jansan A, Forman JL, Rasmussem S, Bangsgoard R, Fledelius HC, et al. Neonatal risk factors for treatmentdemanding retinopathy of prematurity. Ophthalmology [Internet]. 2016 [acesso em 15 jul 2017];123:796-803. Disponível em: http://www.aaojournal.org/article/S0161-6420(15)01488-8/fulltext.

42. Asano MK, Dray PB. Retinopathy of prematurity. Disease-a-Month [Internet]. 2014[acesso em 10 jun 2016];60:282-91. Disponível em: http://www.diseaseamonth.com/article/S0011-5029(14)00046-7/pdf. 
43. Fors MS, Armas MM, Martínez RR, Hernández ML, González YT. Características clínicas epidemiológicas de la retinopatía de la prematuridad en recién nacidos de embarazos múltiples. Revista Cubana de Oftalmología [Internet]. 2013[acesso em 22 mar 2016];26(1):121-8. Disponível em: http://www.revoftalmologia.sld.cu/index.php/oftalmologia/article/view/174/html.

44. Wallace DK, Kylstra JA, Phillips SJ, Hall JG. Poor postnatal weight gain: a risk fator for severe retinopathu of prematurity. J AAPOS[Internet]. 2000[acesso em 22 mar 2016];4:343-7. Disponível em: http://www.jaapos.org/article/S1091-8531(00)105476/pdf.

45. Shah VA, Yeo CL, Ling YL, Ho LY. Incidence, risk factors of retinopathy of prematurity among very low birth weight infants in Singapore. Ann Acad Med Singapore [Internet]. 2005[acesso em 10 mai 2016];34:169-78. Disponível em:

http://www.annals.edu.sg/pdf/34Vol200501/V34N2p169.pdf.

46. Kim TI, Sohn J, Pi SY, Yoon YH. Postnatal risk factors of retinopathy of prematurity. Pediatr Perinat Epidemiol[Internet]. 2004[acesso em 22 mar 2016];18:130-4. Disponível em: http://onlinelibrary.wiley.com/doi/10.1111/j.13653016.2003.00545.x/abstract? systemMessage=Wiley+Online+Library+will+be+unavailable+on+2nd+Dec+2017+starting+from+0800+ $\mathrm{EST}+\% 2 \mathrm{~F}+1300+\mathrm{GMT}+\% 2 \mathrm{~F}+21.00+\mathrm{SGT}+$ for+2.5+hours+due+to+urgent+server+maintenance.+Apologies+for+the+inconvenience.

47. Hartnett ME, Penn JS. Mechanisms and management of retinopathy of prematurity. N EngI J Med [Internet]. 2012 [acesso em 22 mar 2016];367(26):2515-26. Disponível em: http://www.nejm.org/doi/full/10.1056/NEJMra1208129.

48. Ohlsson A, Aher SM. Early erythropoietin for preventing red blood cell transfusion in perterm and/or low birth weight infants. Cochrane Database Syst Ver [Internet]. 2006[acesso em 07 mai 2016];3:CD004863. Disponível em: https://www.ncbi.nlm.nih.gov/pubmed/16856062.

49. Brown MS, Barón AE, France EK, Hamman RF. Association between higher cumulative doses of recombinante erythropoietin and risk factors for retinopathy of prematuriy. J AAPOS [Internet]. 2006[acesso em 07 mai 2016];10:143-9. Disponível em: http://www.jaapos.org/article/S1091-8531(05)00291-0/fulltext.

50. Morita M, Ohneda O, Yamashita T, Takahashi S, Suzuki N, Nakajima O, et al. HLF/HIF-2alpha is a key fator in retinopathy of prematurity in association with erythropoietin. EMBO J [Internet]. 2003[acesso em 07 mai 2016];22:1134-46. Disponível em: https://www.ncbi.nlm.nih.gov/pmc/articles/PMC150350/.

51. Wright KW, Sami D, Thompson L, Ramanathan R, Joseph R, Farzavandi S. A physioloic reduced oxygen decreases the incidence of threshold retinopathy of prematurity. Trans Am Ophthalmol Soc [Internet]. 2006[acesso em 07 mai 2016];104:78-84. Disponível em: https://www.ncbi.nIm.nih.gov/pmc/articles/PMC1809904/.

52. Roberts Jr JD, Fineman JR, Morin FC, Shaul PW, Rimar S, Schreiber MD, et al. Inhaled nitric oxide and persistent pulmonary hypertension of the newborn. The Inhaled Nitric Oxide Study Group. N

53. Mittal M, Dhanireddy R, Higgins RD. Candida sepsis and association with retinopathy of prematurity. Pediatrics[Internet]. 1998[acesso em 07 mai 2016];101:654-657. Disponível em: http://pediatrics.aappublications.org/content/101/4/654.

54. Tomé VAV, Vieira JF, Oliveira LB, Pinto RMC, Abdallah VOS. Estudo da retinopatia da prematuridade em um hospital universitário. Arq Bras Oftalmol [Internet]. 2011[acesso em 05 fev 2016];74(4):279-282. Disponível em:

http://www.scielo.br/pdf/abo/v74n4/v74n4a10.pdf.

55. Hellström A, Smith LEH, Dammann O. Retinopathy of prematurity. Lancet [Internet].2013[acesso em 05 fev 2016];382(9902):1445-1457. Disponível em: https://www.ncbi.nlm.nih.gov/pmc/articles/PMC4389630/.

56. Damman O. Inflamation and retinopathy of prematurity. Acta Pediatr[Internet]. 2010[acesso em 05 fev 2016];99:975-77.

Disponível em: https://www.ncbi.nlm.nih.gov/pmc/articles/PMC2902705/.

57. Sood BG, Madan A, Saha S, Schendel D, Thorsen P, Skogstrand K, et al. Perinatal systemic inflammatory response syndrome and retinopathy of prematurity. Pediatr Res [Internet]. 2010[acesso em 07 mar 2016];67:394-400. Disponível em:

https://www.ncbi.nlm.nih.gov/pmc/articles/PMC2873779/.

58. Chen M, Citil A, McCabe F, Leicht KM, Fiascone J, Dammann CE, et al. Infection, oxygen, and immaturity: interacting risk factors for retinopathy of prematuriy. Neonatology [Internet]. 2011[acesso em 07 mar 2016];99:125-32. Disponível em:

https://www.ncbi.nlm.nih.gov/pmc/articles/PMC2939989/.

59. Behrman RE, Matin CG, Snider Ar, Katz SM, Peabody JL, Brady CJP. Abnormal cerebral blood flow patterns in preterm infants with a large patente ductus arterious. J Pediatr[Internet]. 1982[acesso em 07 mar 2016];101:587-93. Disponível em:

https://www.sciencedirect.com/science/article/pii/S0022347682807154.

60. Brasil. Ministério da Saúde. Atenção à saúde do recém-nascido: Cuidados Gerais. 2 ed. Brasília: DF; 2014. 1:29-50.

61. García Serrano JL, Ramirez Garcia MC, Piñar Molina R. Enfermedad plus en la retinopatía del prematuro de gestación múltiple. Análisis de riesgo. Arch Soc Esp Oftalmol[Internet]. 2009[acesso em 07 mar 2016];84(4):191-8. Disponível em:

http://scielo.isciii.es/pdf/aseo/v84n4/original3.pdf.

62. Gruslin A, Lemayre B. Pre-eclampsia: fetal assessment and neonatal autcomes. Best Pract Res Clin Obstet Gynaecol[nternet]. 2011[acesso em 07 mar 2016];25:491-507. Disponível em:

http://www.sciencedirect.com/science/article/pii/S1521693411000411?via\%3Dihub.

Engl J Med[Internet]. 1997[acesso em 07 mai 2016];336:605-10. Disponível em:

http://www.nejm.org/doi/full/10.1056/NEJM199702273360902. 